\title{
Towards Extraction of Massive DIB Datasets from Stellar Spectroscopic Surveys
}

\author{
R. Lallement ${ }^{1}$, H.C Chen ${ }^{1}$ L. Puspitarini ${ }^{1}$, P. Bonifacio ${ }^{1}$, \\ C. Babusiaux ${ }^{1}$ and V. Hill ${ }^{2}$ \\ 1: GEPI, Paris Observatory Meudon, France, email: rosine.lallement@obspm.fr \\ 2: CASSIOPEE/Cote d'Azur Observatory, Nice, FRANCE
}

\begin{abstract}
Medium to high-resolution stellar spectroscopic surveys can potentially be used to build DIB databases by means of automated methods of analysis. Multiplex spectrographs increase strongly those potentialities and allow small-scale variability studies. Because measurements of the stellar parameters are generally the primary goal of the surveys, synthetic spectra can be computed and used to extract DIBs from late-type star data. Large datasets should allow deeper investigations on the DIB variability in response to stellar radiation fields, DIB reddening relationships, and help localizing interstellar clouds. Here we describe our attempts to build and test automated methods adapted to both early and late type stars.
\end{abstract}

Keywords. ISM, Diffuse Interstellar Bands, Extinction

\section{Introduction}

Several stellar spectroscopic surveys are in progress or in preparation. In particular, the ongoing Gaia ESO Spectroscopic Survey (GES) is a 5 years project at the ESO-VLT in support to the ESA cornerstone astrometric mission Gaia. GES will provide medium and high resolution spectra from which DIBs can be extracted. Given the large diversity of the observed regions and the availability of large numbers of angularly close targets, those data may bring useful statistical information on the links between diffuse interstellar bands (DIBs) and the interstellar environment. DIB equivalent widths can also be used as a complementary tool to other ISM tracers for locating interstellar (IS) clouds. Our goal is to develop fully automated methods of DIB measurements to be applied to such extensive data from the stellar surveys, methods that are adapted for both early and late type stars.

\section{Method for early type star spectra}

An automated DIB fitting method appropriate for early-type stars has been developed and applied to 16 strong DIBs: 4726.8, 4762.6, 4963.9, 5780.4, 5797.1, 5849.8, 6089.8, 6196.0, 6203.0-6204.5, 6269.8, 6283.8, 6379.3, 6445.3, 6613.6, 6660.7, and 6699.3 $\AA$ in high-resolution spectra of $\sim 130$ targets recorded with the ESO FEROS spectrograph (see Puspitarini et al. 2013). The targets are of late-B and A stars and located within $\simeq 400$ pc. It is a first step to simultaneous and entirely automated equivalent widths (EWs) measurements of multiple DIBs in stellar spectra from surveys. Prior to the DIB extraction itself, we established an empirical DIB model by combining DIB average profiles deduced from the most reddened stars (Left of Fig. 1). When weak telluric lines were present, we applied an automated preliminary step of correction, using a telluric transmission model that is fitted in airmass and Doppler shift to the data and used to eliminate those features. The spectra are fitted to a combination of a smooth stellar 

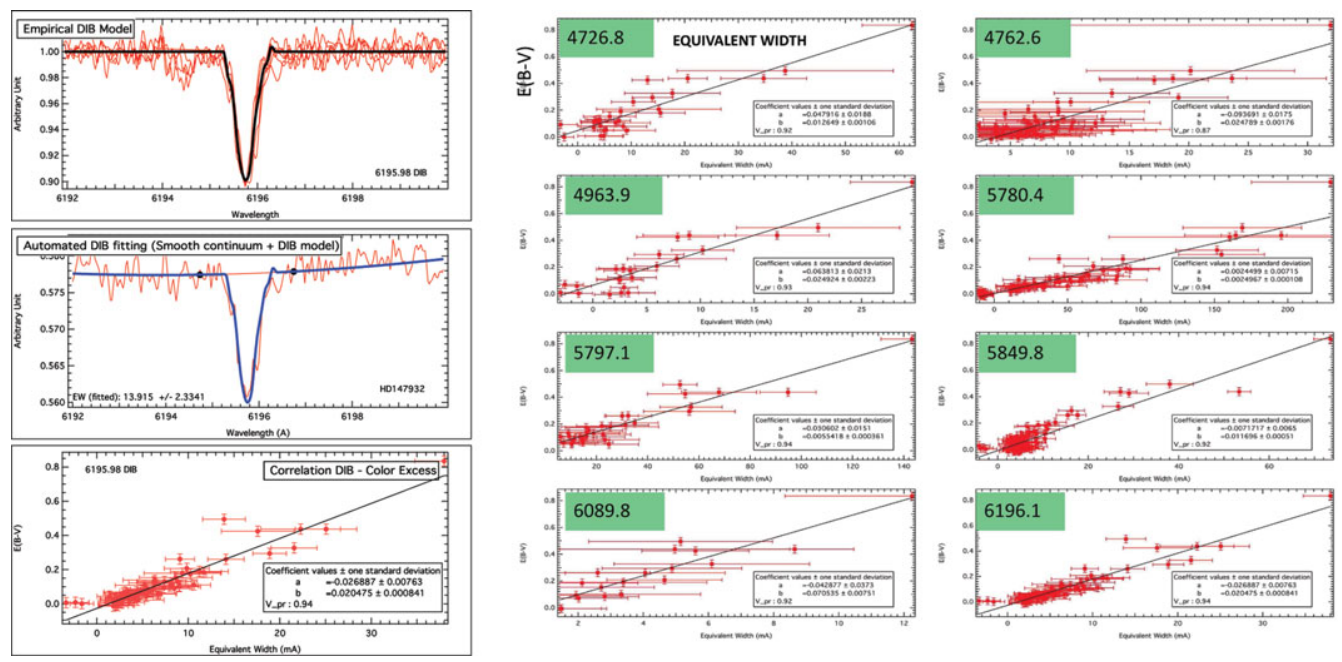

Figure 1. Early-type stars: (left) Empirical DIB profile determination, (middle/right) DIB-reddening relationships for a subset of strong DIBS and nearby late-B/A type stars (from Puspitarini et al. 2013).

continuum represented by a polynomial function and the DIB model (Fig. 1 left). The DIB model is allowed to vary in strength and velocity shift. When strong telluric lines are present, we fit the data to a triple combination of a telluric transmission model, a DIB model, and a smooth continuum.

For each DIB we derived the mean relationship between the equivalent width and the color excess (Fig. 1). The parameters of the linear correlation relationships were positively compared with published values (such as, Friedman et al. 2011, Vos et al. 2011). Most correlation coefficients were found to be higher than those based on earliertype, nearby target stars, which confirms the previous results of (Raimond et al. 2012) based on the same dataset. The reason for this improved correlation is the decrease of the large dispersion linked to the influence of the strong radiation field of the $\mathrm{O}$ or early B stars. First correlation parameters and coefficients were given for the 5849.8, 6089.8, 6269.8, 6445.3, 6660.7, and 6699.3 A DIBs in the Milky Way.

\section{Method for late type star spectra, first DIB-distance relationship}

The method of automated DIB extraction for late-type (cool) star spectra, based on a combination of stellar synthetic, DIB, and telluric transmission models, is detailed by Chen et al. (2013) and Chen et al. (this volume). We show some examples of extractions for two red giants (Fig. 2 top and bottom resp.) in the direction of the galactic bulge, and for the 6284, 6196, and 6203-4 bands (from left to right). For each figure, upper curves are the data (red) and the fit result (blue) while lower curves are the fit components. It can be seen that uncertainties on the DIB EW depend not only on the $\mathrm{S} / \mathrm{N}$ but also on the star radial velocity that influences the location of the strong stellar lines w.r.t. the DIB. It also depends on the metallicity. We illustrate the results for $\simeq 220$ targets from two 12' wide bulge fields observed with VLT/FLAMES/Giraffe by showing in Fig. 3 the relationship between the $6284 \AA$ ADIB equivalent width and a preliminary estimate of the photometric distance. It is compared with the extinction derived by Marshall et al. (2006) based on 2MASS and the Besançon model (black curves), in the $0.25 \times 0.25 \mathrm{deg}^{2}$ 

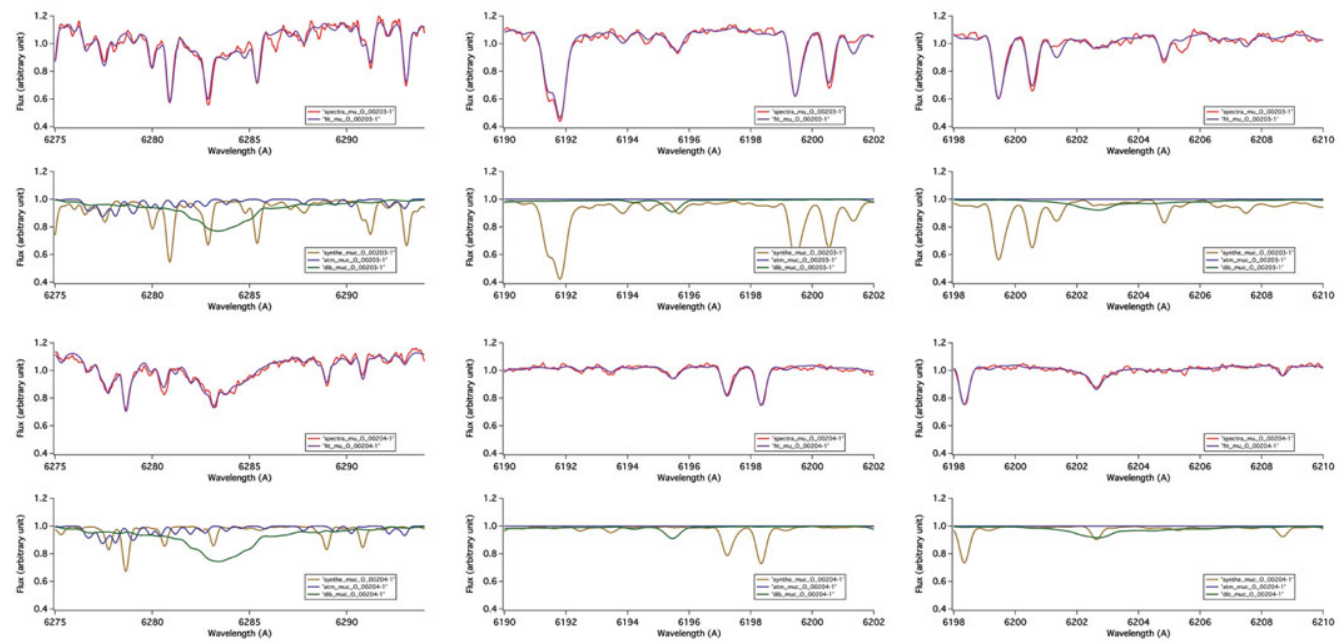

Figure 2. Some cool stars fitting examples: the 6284, 6196, 6203-4 A bands.

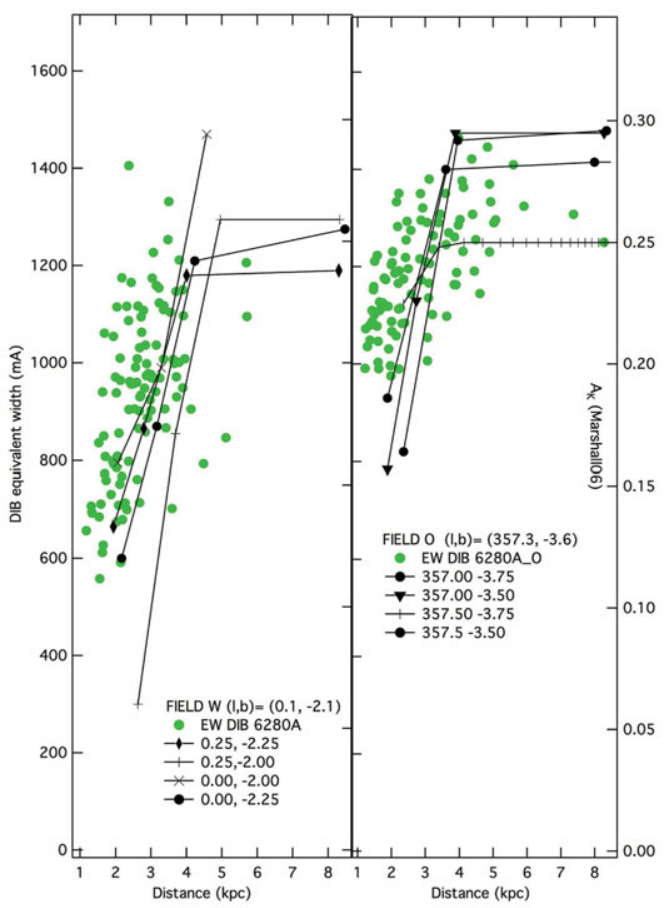

Figure 3. DIB distance relationship.

bins enclosing the field. Both the model and the DIB strength show that most of the extinction is reached at $4 \mathrm{kpc}$ in those directions.

\section{Discussion}

Automated DIB extraction from early- and late-type spectra from stellar spectroscopic surveys appears feasible. Improvements are still needed in various directions: - corrections of the synthetic models for missing or over(under) estimated stellar lines, - automated 
elimination of ambiguous cases and (or) automated derivation of errors associated to the use of the synthetic spectra in each individual case. Finally, allowance for cloud (and DIB) multiplicity must be introduced, especially for the narrowest bands.

\section{Acknowledgements}

We thank financial support from ANR through the STILISM project. H-C C. thanks support from the Region IdF DIM-ACAV.

\section{References}

Chen, H.-C., Lallement, R., Babusiaux, C., et al. 2013, A\&̛A, 550, A62

Friedman, S. D., York, D. G., McCall, B. J., et al. 2011, ApJ, 727, 33

Marshall, D. J., Robin, A. C., Reylé, C., Schultheis, M., \& Picaud, S. 2006,A\&A, 453, 635

Puspitarini, L., Lallement, R., \& Chen, H.-C. 2013, A\&A A, 555, 25

Raimond, S., Lallement, R., Vergely, J. L., Babusiaux, C., \& Eyer, L. 2012, A\&\&A, 544, A136

Vos, D. A. I., Cox, N. L. J., Kaper, L., Spaans, M., \& Ehrenfreund, P. 2011, A\&A, 533, A129 